\title{
Physical Diagnosis: When Blue is a Clue
}

\author{
Matthew A. Murphy, MD
}

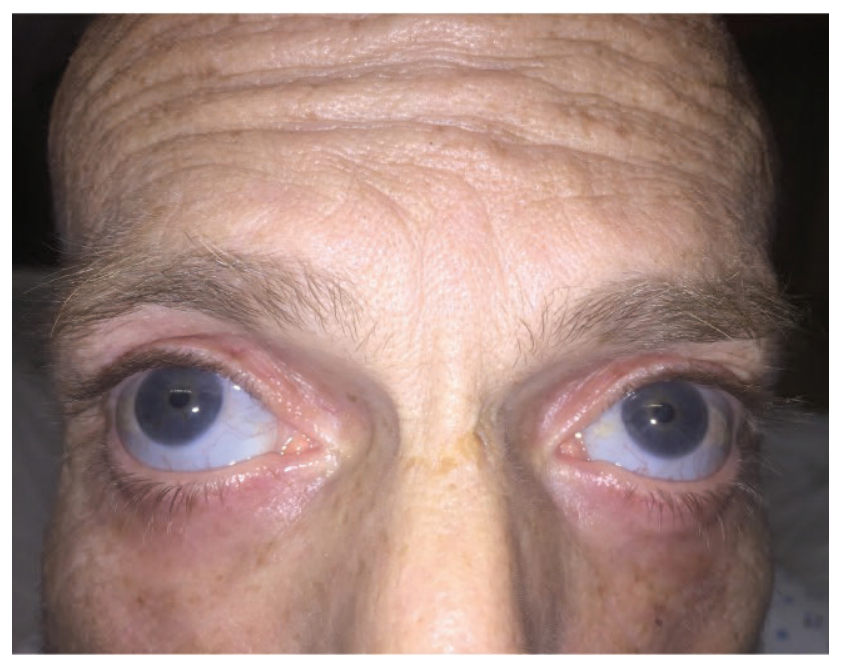

Figure 1. The classic blue sclera of a patient with type 1 osteogenesis imperfecta. Due to a defect in type 1 collagen, the overlying sclera is abnormally thin, resulting in increased visibility of the underlying choroidal veins and the blue color of the eyes. This patient also had a history of more than forty bone fractures throughout his life.

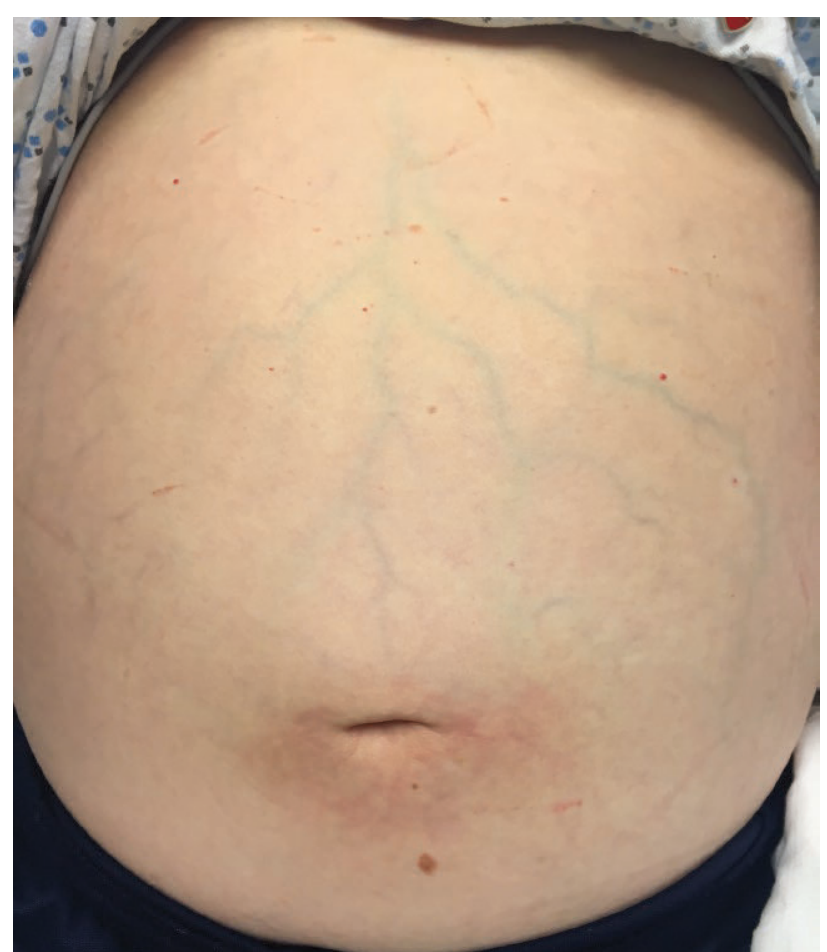

Figure 2. An excellent example of caput medusae in a patient with newly diagnosed cirrhosis. Severe portal hypertension causes shunting of blood through the umbilical vein, which is normally obliterated early in life. This leads to engorgement of the superficial veins of the abdominal wall. 